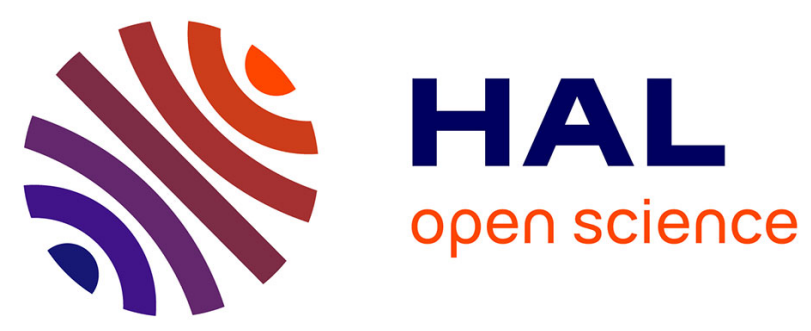

\title{
Filtering, segmentation and region classification by hyperspectral mathematical morphology of DCE-MRI series for angiogenesis imaging
}

Guillaume Noyel, Jesus Angulo, Dominique Jeulin, D. Balvay, C. A. Cuénod

\section{- To cite this version:}

Guillaume Noyel, Jesus Angulo, Dominique Jeulin, D. Balvay, C. A. Cuénod. Filtering, segmentation and region classification by hyperspectral mathematical morphology of DCE-MRI series for angiogenesis imaging. 5th IEEE International Symposium on Biomedical Imaging: From Nano to Macro (ISBI 2008), May 2008, Paris, France. pp.1517-1520, 10.1109/ISBI.2008.4541297 . hal-00834030v2

HAL Id: hal-00834030

https://hal-mines-paristech.archives-ouvertes.fr/hal-00834030v2

Submitted on 23 May 2017

HAL is a multi-disciplinary open access archive for the deposit and dissemination of scientific research documents, whether they are published or not. The documents may come from teaching and research institutions in France or abroad, or from public or private research centers.

$$
\text { Copyright }
$$

L'archive ouverte pluridisciplinaire HAL, est destinée au dépôt et à la diffusion de documents scientifiques de niveau recherche, publiés ou non, émanant des établissements d'enseignement et de recherche français ou étrangers, des laboratoires publics ou privés. 


\section{FILTERING, SEGMENTATION AND REGION CLASSIFICATION BY HYPERSPECTRAL MATHEMATICAL MORPHOLOGY OF DCE-MRI SERIES FOR ANGIOGENESIS IMAGING}

\author{
G. Noyel, J. Angulo, D. Jeulin \\ Centre de Morphologie Mathématique \\ Ecole des Mines de Paris \\ 35 rue Saint Honoré, 77305 Fontainebleau, France
}

\begin{abstract}
Segmenting dynamic contrast enhanced-MRI series of small animal, which are intrinsically noisy and low contrasted images with low resolution, is the aim of this paper. To do this, a segmentation method taking into account the temporal (spectral) and spatial information is presented on several series. The idea is to start from a temporal classification, and to build a probability density function of contours conditionally to this classification. Then, this function is segmented to find potentially tumorous areas. The method is presented on several series after a range normalization histogram in order to compare the series.
\end{abstract}

Index Terms - Hyperspectral images, mathematical morphology, MRI, segmentation, angiogenesis imaging

\section{INTRODUCTION}

The aim of this paper is to segment image series of DCE-MRI (Dynamic Contrast Enhanced MRI) of small animal, using mathematical morphology tools developed for hyperspectral images. The images are series of 512 channels of size $128 \times$ 128 acquired at a regular step of 1 second, in time, on mice presenting tumors [1]. Although the images are time series, we use the terminology of hyperspectral images.

Hyperspectral images are multivariate discrete functions with several tens or hundreds of spectral bands. In a formal way, for each pixel of a 2D hyperspectral image is considered a vector with values in wavelength, time or associated with any index $j$. To each wavelength, time or index corresponds an image in two dimensions called channel. In the sequel, we use the term of spectrum and spectral channel to describe temporal phenomena. Let $\mathbf{f}_{\lambda}: E \rightarrow \mathcal{T}^{L}(x \rightarrow$ $\left.\mathbf{f}_{\lambda}(x)=\left(f_{\lambda_{1}}(x), f_{\lambda_{2}}(x), \ldots, f_{\lambda_{L}}(x)\right)\right)$, be a multispectral image, where: $E \subset \mathbb{R}^{2}, \mathcal{T} \subset \mathbb{R}$ and $\mathcal{T}^{L}=\mathcal{T} \times \ldots \times \mathcal{T}$; $x=x_{i} \backslash i \in\{1,2, \ldots, P\}$ is the spatial coordinates of a vector pixel $\mathbf{f}_{\lambda}\left(x_{i}\right)$ (P is the pixels number of $E$ ); $f_{\lambda_{j}} \backslash j \in$ $\{1,2, \ldots, L\}$ is a channel ( $L$ is the channels number); $f_{\lambda_{j}}\left(x_{i}\right)$ is the value of vector pixel $\mathbf{f}_{\lambda}\left(x_{i}\right)$ on channel $f_{\lambda_{j}}$.

\author{
D. Balvay, C-A. Cuenod
}

\author{
LRI-EA4062 Paris V Descartes \\ APHP, HEGP, Service de Radiologie \\ Paris, France
}
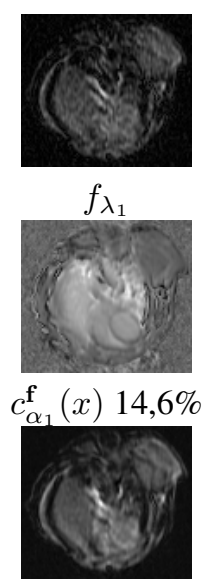

$\widehat{f}_{\lambda_{1}}$
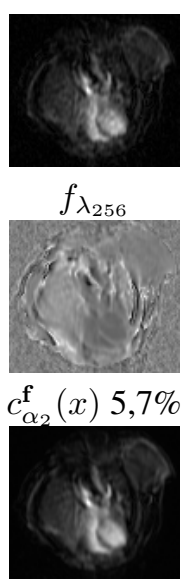

$\widehat{f}_{\lambda_{256}}$
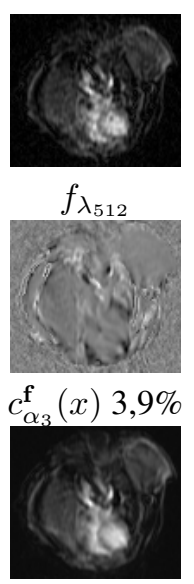

$\widehat{f}_{\lambda_{512}}$
Fig. 1. Representation of channels of the series in various spaces: image space $\mathbf{f}_{\lambda}$ (512 channels), factor space $\mathbf{c}_{\alpha}^{\mathbf{f}}$ (16 channels) with inertias, filtered image space $\widehat{\mathbf{f}}_{\lambda}$ (512 channels)

\section{PRE-PROCESSING}

As shown in [2], Factor Correspondence Analysis (FCA) is useful to filter out the noise in multivariate images. FCA transforms the pixels $\mathbf{f}_{\lambda}\left(x_{i}\right)$ into factor pixels $\mathbf{c}_{\alpha}^{\mathbf{f}}\left(x_{i}\right)$ leading to another hyperspectral image in factor space: $\zeta: \mathcal{T}^{L} \rightarrow$ $\mathcal{T}^{K} / K<L\left(\mathbf{f}_{\lambda}(x) \rightarrow \mathbf{c}_{\alpha}^{\mathbf{f}}(x)=\left(c_{\alpha_{1}}^{\mathbf{f}}(x), \ldots, c_{\alpha_{K}}^{\mathbf{f}}(x)\right)\right)$. After a reconstruction with 16 factorial axes, representing $41 \%$ of inertia, a filtered image is obtained: $\widehat{\mathbf{f}}_{\lambda}(x)=$ $\left(\widehat{f}_{\lambda_{1}}(x), \ldots, \widehat{f}_{\lambda_{L}}(x)\right)$ (fig. 1). A linear model is fitted for each vector pixel, excluding the first transitory twenty channels corresponding to the injection of the contrast agent (fig. $2 \& 3$ ). This model $\widehat{\mathbf{f}}_{\lambda}(x) \sim a(x) \lambda_{j}+b(x)$ with $j \in[21, \ldots, 512]$ has two parameters, the slope $a$ and the intercept $b$. On the first 20 channels, the rise $m$ is defined: $\left.m(x)=\max _{j \in[1: 20]}\left(\widehat{f}_{\lambda_{j}}(x)\right)-\min _{j \in[1: 20]}\left(\widehat{f}_{\lambda_{j}}(x)\right)\right)$. Therefore, a parameter image is created with three channels: $\mathbf{p}(x)=(a(x), b(x), m(x))$.

Consequently, dimensionality reduction is performed either by FCA, or by model approach. Then, the classification 


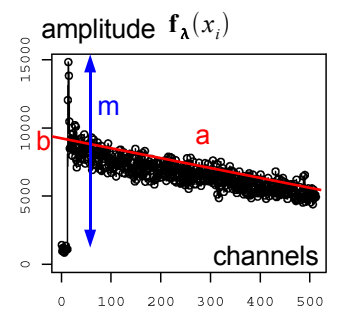

Fig. 2. Model fit at point $(66,62)$ of the heart cavity.

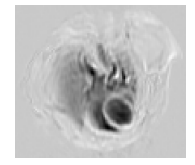

slope $a$

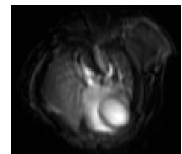

intercept $b$

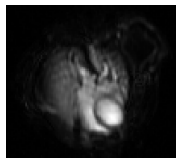

rise $m$
Fig. 3. Parameters maps of the linear model fit for each pixel.

is computed on these spaces of lower dimension.

\section{PIXEL-TEMPORAL CLASSIFICATION}

Classification is performed in the temporal dimension by unsupervised and semi-supervised methods.

For the unsupervised approach, k-means classification in five classes is applied to the factor space $\mathbf{c}_{\alpha}^{\mathbf{f}}$. Another classification is made, according to the minimum distance $L_{1}$ between the linear model of each average filtered spectrum of the k-means classes, and the linear model of each pixel vector of the filtered image $\widehat{f}_{\lambda}$ (fig. 4). This method is called model classification. Both methods are directly applied to other series, and compared to references given by the doctors (fig. 5). The general name of the series is "serimxxx" with "serim447" the initial one and "serim415" and "serim450" the two others.

We notice that the classifications give the limits of the main areas having similar spectra. Besides, the model classification seems to be more robust than the k-means classification. In fact the model decreases the entropy of the image by introducing a prior information for the pixel spectrum.

A semi-supervised classification by Linear Discriminant Analysis (LDA) is also performed on two spaces: the spectrum of the filtered image $\widehat{\mathbf{f}}_{\lambda}$ and of the parameters $\mathbf{p}$ (fig. 6). The training and test errors computed on 80 vector pixels by a 5-fold cross validation are both equal to zero.

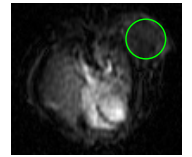

reference

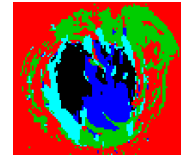

k-means

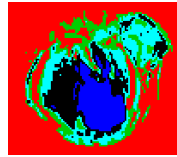

model
Fig. 4. Unsupervised classification in 5 classes. The green circle is the position of the tumor given by the doctors.

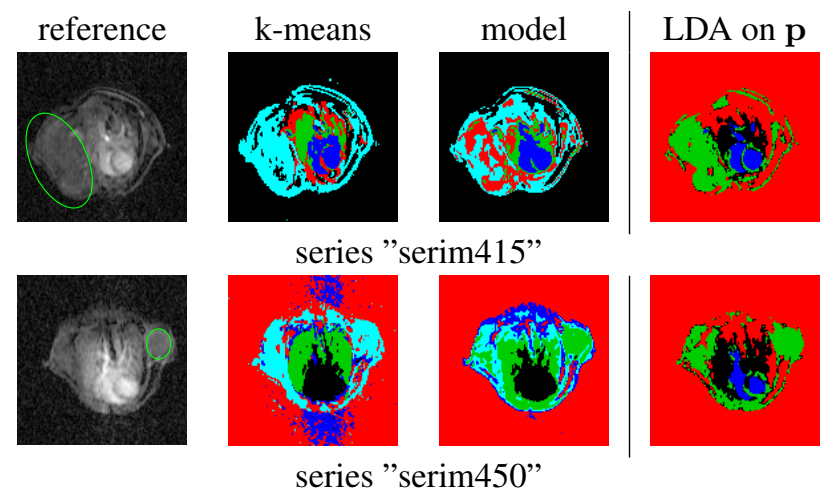

Fig. 5. Unsupervised classification (k-means and model) in 5 classes and semi-supervised classification (LDA) in 4 classes for two different series than in figures 4 and 6 .

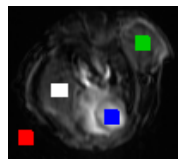

train

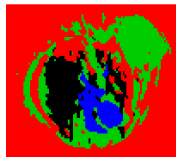

LDA on $\widehat{\mathbf{f}}_{\lambda}$

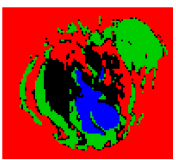

LDA on $\mathbf{p}$
Fig. 6. Semi-supervised classification LDA in 4 classes.

In order to use a LDA on other image series, with the same training pixels, the range of the grey levels of the images must be similar; otherwise the projection of the pixels of these series on the classified space is incoherent. Consequently, we have introduced a range normalization method based on histogram anamorphosis. To get more robust results, the multivariate image of parameters $\mathbf{p}$ is used. For each parameter, the cumulative distribution function (cdf) of the values is estimated. The cdf is the primitive of the density function estimated by an histogram. It is composed of 255 classes defined on parameters of the initial series. The cdf is transformed by a numerical anamorphosis in order to be similar to the cdf of the initial series "serim447" (fig. 7).

After the range normalization of histograms, a LDA with a training on the selected pixels of the initial image is applied to the other series (fig. 5). We notice that classifications give the limits of the main areas having similar spectra than the training set.

As for unsupervised classification, there are some anatomical parts which are classified as the background, in red. In fact, the spatial information is not used at this stage and the pixels of each class may be disconnected.

\section{SPATIAL-TEMPORAL REGIONAL SEGMENTATION}

The spatial and temporal information is introduced with a multivariate gradient obtained by stochastic watershed (WS) with regionalized random balls markers. 
before normalization
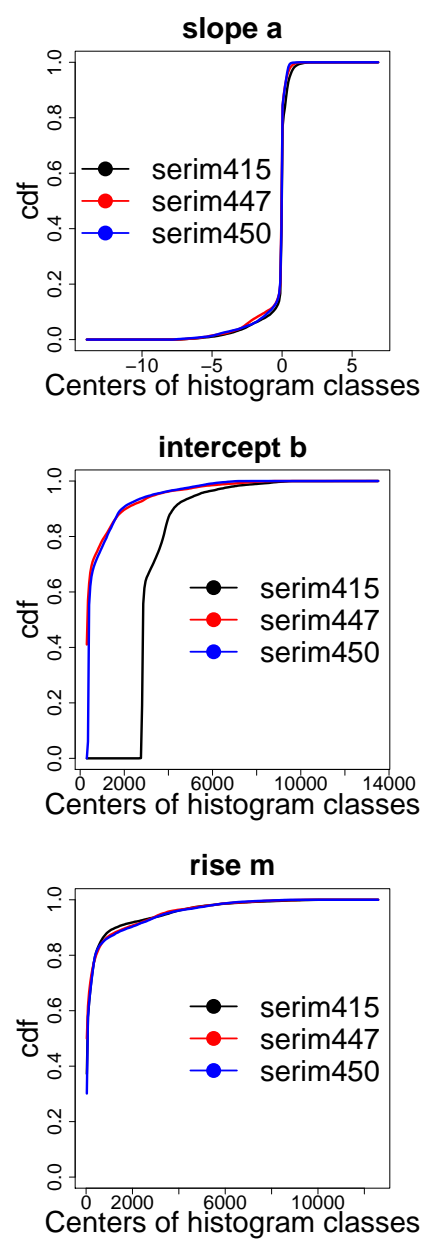

after normalization
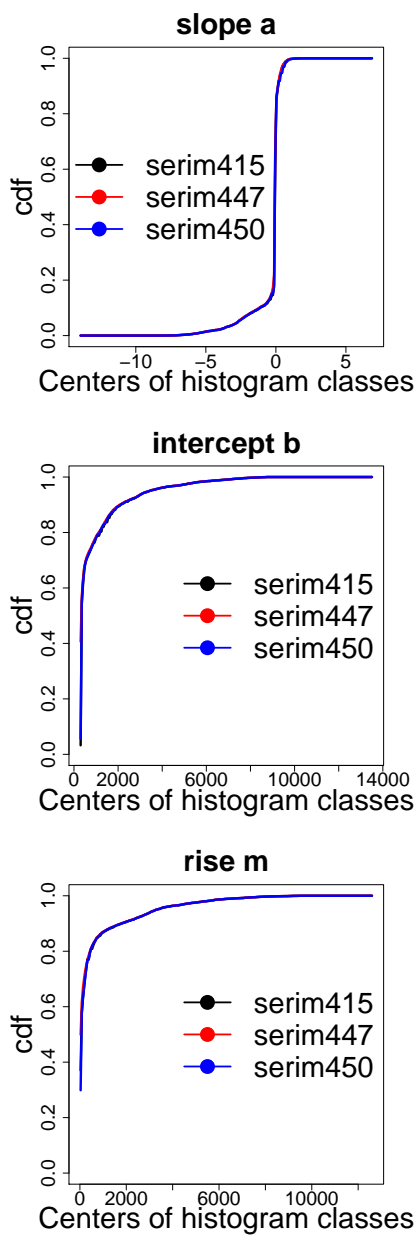

Fig. 7. Cumulative distribution functions computed on the parameters $a, b$ and $m$ of each series before (first column) and after normalization (second column). The reference is the parameters cdf of the initial series "serim447".

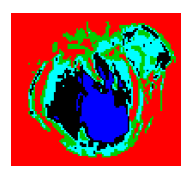

classification

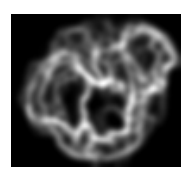

$m p d f$

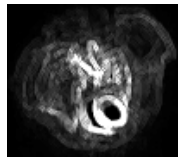

gradient

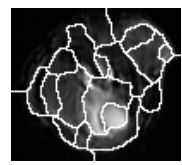

segmentation

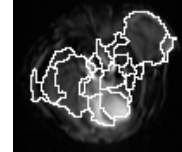

segmentation

Fig. 8. $1^{\text {st }}$ row: stochastic WS starting from the classification by a model, $2^{\text {nd }}$ row: standard WS with the 20 largest regions on volume.

The standard stochastic WS consists in starting from uniform random point markers to flood the norm of a gradient and to obtain associated contours to random markers. After repeating the process a large number of times, a probability density function of contours (pdf) is computed by the Parzen kernel method [3]. The pdf is flooded with a hierarchical watershed according to a volume criterion [4]. For hyperspectral images, a pdf is built for each channel of the image and the flooding function is the weighted sum of the pdf of the channels. This function called a marginal probability density function and contains spatial information [5]. In the sequel, the marginal pdf $m p d f$ is computed conditionally to a temporal classification, using a new way to select markers. Therefore, this pdf contains spatial and temporal information.

Given $\mathcal{D}=\left\{D_{j}\right\}$ a partition, obtained by classification, of disjoint classes of the image space $E \subset \mathbf{R}^{2}$. Each class $D_{j}$ of the partition is composed of connected components $C_{i}$ : i.e. $D_{j}=\cup_{i} C_{i}$. Then the markers $m$ are drawn conditionally to the connected components $C_{i}$ of the classification. To do this, the following rejection method is used: the markers are distributed with a uniform random drawn. If a marker $m$ is inside a connected component $C_{i}$ of minimum area $S$, and not yet marked, then it is kept, otherwise it is rejected. These markers are called regionalized random markers.

Moreover to decrease the probability of small, textured and low contrasted contours regions, we use random balls as markers. The centers of the balls are the regionalized random markers and the radii $r$ are uniformly distributed between 0 and a maximum radius $R \max : \mathcal{U}[1, R \max ]$. Only the intersection between the ball $B(m, r)$ and the connected component $C_{i}$ is kept as marker. These balls are called regionalized random balls markers (algorithm 1).

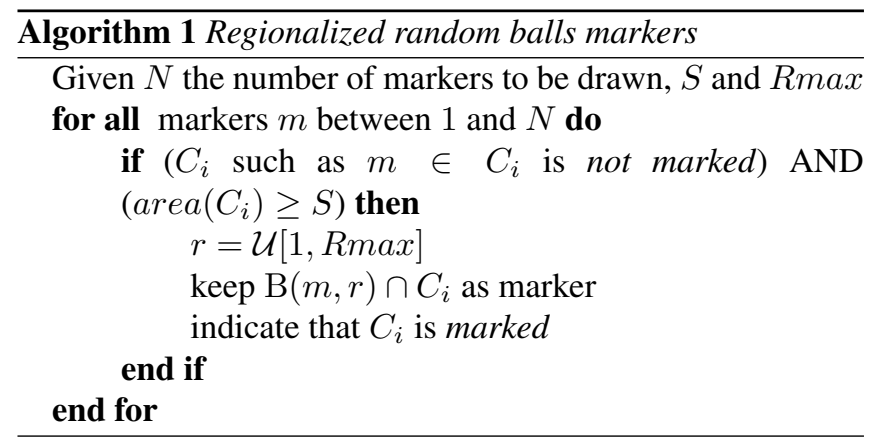

Starting from the classification by a model, which seems more robust, a segmentation is performed by stochastic WS with $N=100$ points, $M=100$ realizations, $R=20$ regions, a minimum area $S=10$ pixels and a maximum radius for random balls of $\operatorname{Rmax}=30$ pixels (fig. 8). The number of regions must be sufficiently high to detect enough contours. With the segmentation, we have tried to detect potentially tumorous areas.

This result is better than a standard hierarchical WS, with the 20 largest regions on a volume criterion, applied on a Ma- 
halanobis distance based-gradient [2] (fig. 8).

\section{COMPUTER-AIDED DETECTION}

Potentially tumorous areas are detected with (1) a positive mean slope parameter $a$, because the contrast agent tends to accumulate in these areas, and (2) a mean intercept $b$ higher than 800 after histogram normalization. This last criterion was empirically defined. Moreover for each area, coefficients of variation are measured. These coefficients $\beta$ are defined as the ratio between the standard deviation $\sigma$ and the mean of the parameter mean for the considered region: $\beta_{a}=\sigma_{a} /$ mean $_{a}$, $\beta_{b}=\sigma_{b} /$ mean $_{b}$. Therefore confidence maps are created for parameters with an adapted color scale for each parameter, starting from the highest confidence in blue to the lowest in red. We notice that for each series, the areas corresponding to tumors have parameters with a higher confidence (fig. 9 (a)). These preliminary results, obtained on 25 series, are very promising, but should be confirmed on more data for further applications.

The whole analysis flowchart is presented on figure 9 (b).

\section{CONCLUSIONS AND PERSPECTIVES}

In this paper, the segmentation of DCE-MRI series by stochastic multivariate watershed with regionalized random balls markers is presented to improve the results obtained by classification. The originality of this approach is to combine spatial and temporal information in a multivariate gradient $m p d f$. Temporal information comes from a classification, which conditions the random generations of the markers for the $m p d f$. Therefore the stochastic watershed is very useful to detect the low contrasted regions corresponding to tumors as it regularizes the contours. Moreover, a range normalization histogram is also tested on parameters in order to obtain similar parameters range. Finally, a computer-aided detection of potentially tumorous areas is proposed, and seems very promising for very difficult data sets.

\section{REFERENCES}

[1] D. Balvay, F. Frouin, G. Calmon, B. Bessoud, E. Kahn, N. Siauve, O. Clément, and C.A. Cuenod, "New criteria for assessing fit quality in dynamic contrast-enhanced $T_{1}$ weighted MRI for perfusion and permeability imaging," Magnetic Resonance in Med., vol. 54, pp. 868-877, 2005.

[2] G. Noyel, J. Angulo, and D. Jeulin, "Morphological segmentation of hyperspectral images," Image Analysis and Stereology, vol. 26, pp. 101-109, November 2007.

[3] J. Angulo and D. Jeulin, "Stochastic watershed segmentation," in Proc. of the 8th Int. Symposium on Mathematical Morphology. MCT/INPE, 2007, vol. 1, pp. 265-276.
[4] F. Meyer, "An overview of morphological segmentation," Int. Jour. Pattern Recognition Artificial Intelligence, vol. 15 (7), pp. 1089-1118, 2001.

[5] G. Noyel, J. Angulo, and D. Jeulin, "Random germs and stochastic watershed for unsupervised multispectral image segmentation," in Proc. KES 2007/ WIRN 2007. LNAI 4694 Springer, 2007, vol. III, pp. 17-24. 

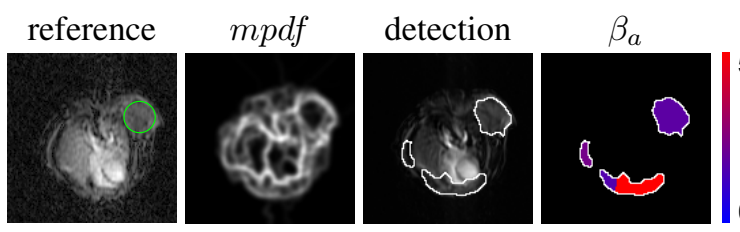

"serim447"
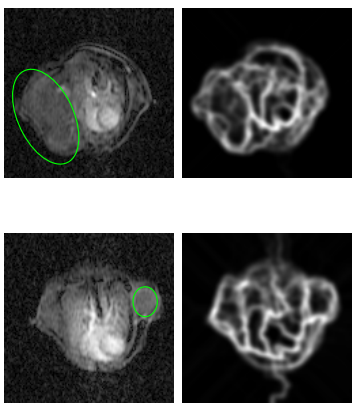

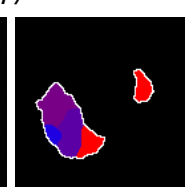

"serim415"

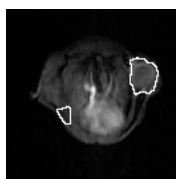

"serim450"

(a) Results

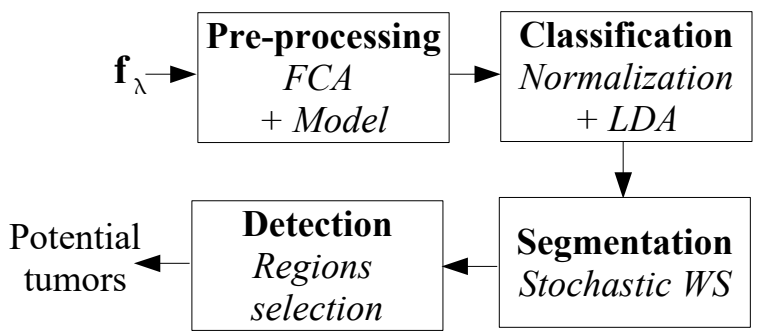

(b) Flowchart of the analysis

Fig. 9. (a) References, $m p d f$, detections of potentially tumorous areas and confidence maps $\beta_{a}$ and $\beta_{b}$ on parameters for the three series. (b) Flowchart od the whole analysis pipeline. 